\title{
A Novel Mutation in ATP7B Gene Associated with Severe Neurological and Psychiatric Symptoms
}

\author{
V. Mihaylova ${ }^{a}$ T. Todorov ${ }^{b} \quad$ I. Tournev ${ }^{a}$ S. Cherninkova ${ }^{a} \quad$ N. Nikoevski ${ }^{a} \quad$ M. Raicheva ${ }^{a}$ \\ P. lankova ${ }^{a}$ I. Petrova ${ }^{a}$ A. Savov ${ }^{b}$ I. Kremesky ${ }^{b}$ \\ ${ }^{a}$ Department of Neurology, University Hospital Alexandrovska, and ${ }^{b}$ Laboratory of Molecular Pathology, \\ Medical University Sofia, Sofia, Bulgaria
}

Dear Sir,

Wilson's disease is a relatively rare inherited autosomal recessive disorder of copper metabolism that results in accumulation of copper in the liver and subsequently in other organs, mainly the central nervous system $[1,2]$. The Wilson's disease gene (ATP7B) encodes a 1,465 amino acid protein of the P-type adenosine triphosphate (ATP) family [3-5, 6]. Absent or reduced function of the ATP7B protein leads to decreased hepatocellular excretion of copper into bile and failure of its incorporation into ceruloplasmin $[3,6,7]$. It contains six copper-binding regions, an ATP domain, a transmembrane cation channel and an actuator domain. The ATP-binding domain consists of the phosphorylation domain ( $\mathrm{P}$ domain) and the nucleotide-binding domain ( $\mathrm{N}$ domain). The $\mathrm{P}$ domain and the actuator domain play an essential role in ATP7B functioning. It is not surprising that these two domains contain the sequence motifs found in all P-type ATPases. The $\mathrm{N}$ domain is equally important and has a number of invariant residues within the $\mathrm{P}_{1}$-ATPase subgroup. Such residues are His-1069 and Glu-1064, which have been shown to be important for ATP binding [5].

The gene for Wilson's disease was first reported in 1993, and since then at least 200 different disease-specific mutations have been described. The most common mutation in European populations is H1069Q $[3,4]$.

Wilson's disease occurs worldwide with an average prevalence of 1:30,000 [3,7]. Signs and symptoms of Wilson's disease are rarely observed before the age of 3 years [3, 7]. Although the upper age limit for consideration of Wilson's disease is generally stated as less than 40 years, the oldest patients with confirmed diagnosis by molecular studies are in their eighth decade [7]. Patients present typically with liver, neurological or psychiatric symptoms, sometimes with a combination of these [1-3, 7]. Approximately $45 \%$ of all affected individuals present with liver disease, $35 \%$ with neurological signs and symptoms and $10 \%$ with psychiatric disturbances [3]. The remaining $10 \%$ present with hemolytic anemia, cardiomyopathy and a number of other less common features such as renal and skeletal abnormalities, pancreatitis, hypoparathyroidism, infertility or repeated miscarriages $[3,7]$. Neurological presentation of Wilson's disease include tremor, incoordination, drooling, dysarthria, dystonia, parkinsonian symptoms, micrographia $[1,2,7]$. Along with behavioral changes, other psychiatric manifestations are depression, anxiety, reduced sexual inhibition or frank psychosis [2, 7]. Kayser-Fleischer rings, arising from copper deposition at Descemet's membrane, are almost always present in patients with neurological symptoms $[1,2,7]$. The diagnosis of Wilson's disease is determined by the presence of the signs and symptoms noted previously, in conjunction with laboratory testing that indicates impaired hepatic copper metabolism [2, 3, 7]. Because of the fact that Wilson's disease is quite effectively treated, early recognition and diagnosis of the disease is of paramount importance [1, 2, 7].

We report on a patient who was a compound heterozygote for a novel mutation in ATP7B and who had a late onset of severe neurological and psychiatric symptoms.

\section{Case Report}

A 49-year-old woman was admitted to our Department with an 8-month history of tremor in the upper limbs more pronounced in the right arm, which gradually increased. Three months later tremor also developed in the lower limbs and in the head. In addition, rigidity in the right extremities and ataxic gait appeared. She had no other disorders except for medically treated diabetes type 2 . The family history was unremarkable.

At our Department, physical and neurological examinations were performed and routine hematological and biochemical studies as well as measurement of serum ce-

\section{KARGER}

Fax +4161306 1234 E-Mail karger@karger.ch www.karger.com
C 2006 S. Karger AG, Basel 0014-3022/06/0552-0099\$23.50/0

Accessible online at: www.karger.com/ene
Violeta Mihaylova

Luilin bl. 519, vh.2, app.15

BG-1359 Sofia (Bulgaria)

Tel. +359 22548 83, Fax +35929230672

E-Mailvioletamihaylova@yahoo.com 
ruloplasmin, serum copper and urinary copper excretion were done. Ultrasound scanning of the abdomen as well as the heart and CT and MRI of the brain were performed. Neuropsychological testing and slit-lamp examination were also carried out. The tremor was recorded by EMG. Finally DNA analysis of ATP7B was completed.

Physical examination was normal. On neurological examination, severe cerebellar ataxia presented with ataxic gait, titubation, intention tremor and dysmetria on knee-toheel and finger-to-nose testing; postural tremor and scanning dysarthria were found. In addition, rigidity in the limbs, more prominent in the right ones and cogwheel phenomenon in the right wrist were noted. In both hands there was resting tremor. Neuropsychological testing revealed a mild decrease in the global cognitive functioning (MMSE $=26$, Raven at the lower limits of normal) and pronounced impairment of the executive functions. The following data were recorded: working memory $70 \%$, delayed memory $60 \%$; recognition $100 \%$, and significantly reduced verbal fluency.

Routine hematological and biochemical investigations were within the normal ranges except for the slightly elevated $\gamma$-glutamil transpeptidase. The levels of serum ceruloplasmin measured either enzymatically or immunologically were low: $3 \mathrm{U} / 1$ (normal $>15 \mathrm{U} / \mathrm{l}$ ) and $0.11 \mathrm{~g} / \mathrm{l}$ (normal $>0.15 \mathrm{~g} / \mathrm{l}$ ), respectively. Consistent with this, the concentration of the total serum copper was also decreased $(5.6 \mu \mathrm{mol} / 1$; reference ranges: 11-22 $\mu \mathrm{mol} / \mathrm{l})$. The basal 24hour urinary copper excretion was greater than normal $(2.4 \mu \mathrm{mol} / 24 \mathrm{~h}$; reference limits: $<1.1 \mu \mathrm{mol} / 24 \mathrm{~h}$ ). After $d$-penicillamine challenge test, the amount of urinary copper excretion was approximately 5 times greater (11.44 $\mu \mathrm{mol} / 24 \mathrm{~h})$.

On slit-lamp examination, not fully developed Kayser-Fleischer ring in the inferior part of the cornea of both eyes was observed.

Both the CT and the MRI of the brain showed severe generalized cerebral atrophy. The lateral ventricles were enlarged and the cerebral sulci widened. No signal abnormalities on $\mathrm{T}_{1}$ - or $\mathrm{T}_{2}$-weighted magnetic resonance images were found in the lentiform nuclei, caudate nuclei, thalami, brainstem and dentate nuclei.
On EMG, resting tremor with a frequency of $5 \mathrm{~Hz}$ and amplitude of $400 \mu \mathrm{V}$ from $\mathrm{m}$. opponens pollicis was recorded.

The ultrasound of the liver revealed steatotic changes. The ECG and echocardiography were normal.

After obtaining informed consent from the patient, a genomic DNA was isolated from the peripheral blood by the standard salt procedure. Molecular genetic studies using SSCP analysis screened for mutations in all 21 exons of ATP7B, and the exons differing in migration patterns from control subjects were subjected to direct sequencing. The patient was found to be a compound heterozygote carrying the wellknown H1069Q mutation and the novel D1279Y missense mutation in exon 18.

Treatment with $d$-penicillamine and vitamin $B_{6}$ was started. Four days later, facial rash occurred. Testing for sensitivity to $d$ penicillamine was positive. Penicillamine was discontinued and trientine was administered. Because of financial difficulties she was prevented from proper treatment. Zinc was the only medication she had. Two years after the onset of the tremor she experienced an acute psychotic episode. Several months later the patient died.

\section{Discussion}

The clinical symptoms, neurological examination, laboratory findings, neuroimaging studies as well as DNA analysis are in accord with the diagnosis of Wilson's disease.

It is considered that the genotype-phenotype correlations in Wilson's disease are hampered by the large number of compound heterozygotes [3, 7]. Nevertheless, the results of a recently published metaanalysis of 577 Wilson's disease patients demonstrate that homozygotes or heterozygotes for H1069Q have a late onset of neurological presentation [8]. This correlation might be due to the reduced activity of the ATP7B protein still allowing some excretion of copper [8]. It has been demonstrated that H1069Q mutant's affinity for ATP is reduced at least 15 -fold, while, for example, the substitution of the conserved Glu-1064 results in a complete loss of nucleotide binding [5].

Although there is a large body of evidence that additional factors may signifi- cantly influence both the time of onset and the mode of presentation, for example the apoE genotype [9] or the MURR1 gene [10], we suggest that in our case the novel detected mutation D1279Y contributes to the severe neurological and psychiatric symptoms. The later onset may be attributed to the presence of $\mathrm{H} 1069$ Q.

The novel mutation results in a substitution of Asp for Tyr and in losing the charge of the residue at position 1279. This is likely to cause the $\mathrm{P}$ domain either to malfunction or to not work at all.

It is necessary for our suggestions to be reproduced by other relevant studies. If the mutation D1279Y is detected in homozygous state it will help further elucidate its phenotypic consequences.

\section{References}

1 El-Youssef M: Wilson disease. Mayo Clin Proc 2003; 78:1126-1136.

2 Brewer GJ: Recognition, diagnosis and management of Wilson's disease. Proc Soc Exp Biol Med 2000;223:36-46.

3 Gitlin J: Wilson disease. Gastroenterology 2003; 125:1868-1877.

4 Riordan S, Williams R: The Wilson's disease gene and phenotypic diversity. J Hepatol 2001; 34:165-171.

5 Morgan C, Tsivkovski T, Kosinsky Y, Efremov R, Lutsenko S: The distinct functional properties of the nucleotide-binding domain of ATP7B, the human copper-transporting ATPase. J Biol 2004;279:36363-36371.

6 Kunihiko T, Schilsky M, Naoyuki M, Toshihiro S: ATP7B (WND) protein. Int J Biochem Cell Biol 1998;30:1063-1067.

7 Roberts E, Schilsky M: A practice guideline on Wilson disease. Hepatology 2003;37:14751492.

8 Stapelbroek J, Bollen C, van Amstel P, van Erpecum $\mathrm{K}$, van Hattum J, van den Berg L, Klomp L, Houwen R: The H1069Q mutation in ATP7B is associated with late and neurologic presentation in Wilson disease: results of a meta-analysis. J Hepatol 2004;41:758-763.

9 Stuehler B, Reichert J, Stremmel W, Schaefer $\mathrm{M}$ : Analysis of the human homologue of the canine copper toxicosis gene MURR1 in Wilson disease patients. J Mol Med 2004;82:629634.

10 Schiefermeier M, Kollegger H, Madl C, Oder W, Kuhn J, Berr F, Ferenci P: The impact of apolipoprotein $\mathrm{E}$ genotypes on age at onset of symptoms and phenotypic expression in Wilson's disease. Brain 2000;123:585-590. 